International Journal of Pure and Applied Mathematics

Volume 112 No. 3 2017, 631-643

ISSN: 1311-8080 (printed version); ISSN: 1314-3395 (on-line version)

url: http://www.ijpam.eu

doi: 10.12732/ijpam.v112i3.14

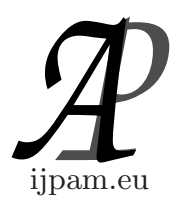

\title{
RELATIONSHIPS BETWEEN $L$-NEIGHBORHOOD SYSTEMS AND L-FUZZY TOPOLOGIES
}

\author{
Jung Mi Ko${ }^{1}$, Ju-Mok $\mathrm{Oh}^{2} \S$ \\ ${ }^{1,2}$ Department of Mathematics \\ Gangneung-Wonju University \\ Gangneung, Gangwondo, 210-702, KOREA
}

\begin{abstract}
In this paper, we investigate two $L$-neighborhood systems induced by an $L$ fuzzy topology in a complete residuated lattice $L$. We study the relationships among $L$-fuzzy topology, $L$-topologies and $L$-neighborhood systems. Finally, we give their examples.
\end{abstract}

AMS Subject Classification: 03E72, 06A15, 06F07, 54A05

Key Words: complete residuated lattice, $L$-neighborhood system, $L$-(fuzzy) topological space

\section{Introduction}

Hájek [6] introduced a complete residuated lattice which is an algebraic structure for many valued logic. It is an important mathematical tool for algebraic structures $[1,7,12]$.

Many researcher introduced the notion of $L$-fuzzy topological structures in unit interval $[0,1]([2,8])$, complete distributive lattices $([4,5,9])$, commutative unital quantales and complete quasi-monoidal lattices $([3,7,12])$. Ramadan et al.[10,11] investigated the relationships between $L$-fuzzy quasi-uniform structures and $L$-fuzzy topological structures in a complete residuated lattice.

\footnotetext{
Received: $\quad$ May 18, 2016

Revised: February 8, 2017

Published: $\quad$ February 9, 2017

(c) 2017 Academic Publications, Ltd. url: www.acadpubl.eu

${ }^{\S}$ Correspondence author
} 
In this paper, we investigate two $L$-neighborhood systems induced by an $L$ fuzzy topology in a complete residuated lattice $L$. We study the relationships among $L$-fuzzy topology, $L$-topologies and $L$-neighborhood systems. Finally, we give their examples.

\section{Preliminaries}

Definition 2.1. $[1,6,7]$ An algebra $(L, \wedge, \vee, \odot, \rightarrow, 0,1)$ is called a complete residuated lattice if it satisfies the following conditions:

(C1) $(L, \leq, \vee, \wedge, 0,1)$ is a complete lattice with the greatest element 1 and the least element 0 ;

(C2) $(L, \odot, 1)$ is a commutative monoid;

(C3) $x \odot y \leq z$ iff $x \leq y \rightarrow z$ for $x, y, z \in L$.

In this paper, we assume that $\left(L, \vee, \wedge, \odot, \rightarrow,^{*}, 1,0\right)$ be a complete residuated lattice with a strong negation *. For $\alpha \in L, \lambda \in L^{X}$, we denote $(\alpha \rightarrow$ $\lambda),(\alpha \odot \lambda), \alpha_{X} \in L^{X}$ as $(\alpha \rightarrow \lambda)(x)=\alpha \rightarrow \lambda(x),(\alpha \odot \lambda)(x)=\alpha \odot \lambda(x)$, $\alpha_{X}(x)=\alpha$.

Lemma 2.2. $[1,6,7]$ Let $\left(L, \vee, \wedge, \odot, \rightarrow,{ }^{*}, 1,0\right)$ be a complete residuated lattice with a strong negation *. For each $x, y, z, x_{i}, y_{i} \in L$, the following properties hold.

(1) If $y \leq z$, then $x \odot y \leq x \odot z$.

(2) If $y \leq z$, then $x \rightarrow y \leq x \rightarrow z$ and $z \rightarrow x \leq y \rightarrow x$.

(3) $x \rightarrow y=1$ iff $x \leq y$.

(4) $x \rightarrow 1=1$ and $1 \rightarrow x=x$.

(5) $x \odot y \leq x \wedge y$.

(6) $x \odot\left(\bigvee_{i \in \Gamma} y_{i}\right)=\bigvee_{i \in \Gamma}\left(x \odot y_{i}\right)$ and $\left(\bigvee_{i \in \Gamma} x_{i}\right) \odot y=\bigvee_{i \in \Gamma}\left(x_{i} \odot y\right)$.

(7) $x \rightarrow\left(\bigwedge_{i \in \Gamma} y_{i}\right)=\bigwedge_{i \in \Gamma}\left(x \rightarrow y_{i}\right)$ and $\left(\bigvee_{i \in \Gamma} x_{i}\right) \rightarrow y=\bigwedge_{i \in \Gamma}\left(x_{i} \rightarrow y\right)$. $\left.y_{i}\right)$.

(8) $\bigvee_{i \in \Gamma} x_{i} \rightarrow \bigvee_{i \in \Gamma} y_{i} \geq \bigwedge_{i \in \Gamma}\left(x_{i} \rightarrow y_{i}\right)$ and $\bigwedge_{i \in \Gamma} x_{i} \rightarrow \bigwedge_{i \in \Gamma} y_{i} \geq \bigwedge_{i \in \Gamma}\left(x_{i} \rightarrow\right.$

(9) $(x \rightarrow y) \odot x \leq y$ and $(x \rightarrow y) \odot(y \rightarrow z) \leq(x \rightarrow z)$.

(10) $x \rightarrow y \leq(y \rightarrow z) \rightarrow(x \rightarrow z)$ and $x \rightarrow y \leq(z \rightarrow x) \rightarrow(z \rightarrow y)$.

(11) $\bigwedge_{i \in \Gamma} x_{i}^{*}=\left(\bigvee_{i \in \Gamma} x_{i}\right)^{*}$ and $\bigvee_{i \in \Gamma} x_{i}^{*}=\left(\bigwedge_{i \in \Gamma} x_{i}\right)^{*}$.

(12) $(x \odot y) \rightarrow z=x \rightarrow(y \rightarrow z)=y \rightarrow(x \rightarrow z)$ and $(x \odot y)^{*}=x \rightarrow y^{*}$.

(13) $x^{*} \rightarrow y^{*}=y \rightarrow x$ and $(x \rightarrow y)^{*}=x \odot y^{*}$.

(14) $y \rightarrow z \leq x \odot y \rightarrow x \odot z$.

$(15)(x \rightarrow y) \odot(z \rightarrow w) \leq(x \odot z) \rightarrow(y \odot w)$, 
Definition 2.3. [1,4,9] Let $X$ be a set. A mapping $R: X \times X \rightarrow L$ is called an $L$-partial order if it satisfies the following conditions:

(E1) reflexive if $R(x, x)=1$ for all $x \in X$,

(E2) transitive if $R(x, y) \odot R(y, z) \leq R(x, z)$, for all $x, y, z \in X$,

(E3) antisymmetric if $R(x, y)=R(y, x)=1$, then $x=y$.

Lemma 2.4. $[1,4,9]$ For a given set $X$, define a binary mapping $S: L^{X} \times$ $L^{X} \rightarrow L$ by

$$
S(\lambda, \mu)=\bigwedge_{x \in X}(\lambda(x) \rightarrow \mu(x)) .
$$

Then, for each $\lambda, \mu, \rho, \nu \in L^{X}$, and $\alpha \in L$, the following properties hold.

(1) $S$ is an $L$-partial order on $L^{X}$.

(2) $\lambda \leq \mu$ iff $S(\lambda, \mu) \geq 1$,

(3) If $\lambda \leq \mu$, then $S(\rho, \lambda) \leq S(\rho, \mu)$ and $S(\lambda, \rho) \geq S(\mu, \rho)$,

(4) $S(\lambda, \mu) \odot S(\nu, \rho) \leq S(\lambda \odot \nu, \mu \odot \rho)$ and $S(\lambda, \mu) \odot S(\nu, \rho) \leq S(\lambda \oplus \nu, \mu \oplus \rho)$,

(5) $S(\mu, \rho) \odot S(\lambda, \mu) \leq S(\lambda, \rho)$ and $S(\mu, \rho) \leq S(\rho, \lambda) \rightarrow S(\mu, \lambda)$,

(6) $\bigvee_{\mu \in L^{X}}(S(\mu, \rho) \odot S(\lambda, \mu))=S(\lambda, \rho)$.

(7) If $\phi: X \rightarrow Y$ is a map, then for $\lambda, \mu \in L^{X}$ and $\rho, \nu \in L^{Y}$,

$$
\begin{aligned}
& S(\lambda, \mu) \leq S\left(\phi^{\rightarrow}(\lambda), \phi^{\rightarrow}(\mu)\right), \\
& S(\rho, \nu) \leq S\left(\phi^{\leftarrow}(\rho), \phi^{\leftarrow}(\nu)\right),
\end{aligned}
$$

and the equalities hold if $\phi$ is bijective.

Definition 2.5. [7,12] A map $\mathcal{T}: L^{X} \rightarrow L$ is called an $L$-fuzzy topology on $X$ if it satisfies the following conditions:

(T1) $\mathcal{T}\left(0_{X}\right)=\mathcal{T}\left(1_{X}\right)=1$

(T2) $\mathcal{T}(\lambda \odot \mu) \geq \mathcal{T}(\lambda) \odot \mathcal{T}(\mu), \quad \forall \lambda, \mu \in L^{X}$,

(T3) $\mathcal{T}\left(\bigvee_{i} \lambda_{i}\right) \geq \bigwedge_{i} \mathcal{T}\left(\lambda_{i}\right), \quad \forall\left\{\lambda_{i}\right\}_{i \in \Gamma} \subseteq L^{X}$

The pair $(X, \mathcal{T})$ is called an $L$-fuzzy topological space. An $L$-fuzzy topological space is called enriched if

(R) $\mathcal{T}(\alpha \odot \lambda) \geq \mathcal{T}(\lambda)$ for all $\lambda \in L^{X}$ and $\alpha \in L$.

Let $\left(X, \mathcal{T}_{1}\right)$ and $\left(Y, \mathcal{T}_{2}\right)$ be two $L$-fuzzy topological spaces. A mapping $\phi$ : $X \rightarrow Y$ is said to be $L$-fuzzy continuous iff for each $\lambda \in L^{Y}, \mathcal{T}_{2}(\lambda) \leq \mathcal{T}_{1}\left(\phi^{\leftarrow}(\lambda)\right)$.

Remark 2.6. [7] A set $\tau \subset L^{X}$ is called an $L$-topology on $X$ if (t1) $0_{X}, 1_{X} \in \tau,(\mathrm{t} 2)(\lambda \odot \mu) \in \tau$, for each $\lambda, \mu \in \tau$, (t3) $\bigvee_{i} \lambda_{i} \in \tau$, for all $\lambda_{i} \in \tau$. An $L$-topology $\tau$ is called enriched if $\alpha \odot \lambda \in \tau$, for all $\lambda \in \tau$ and $\alpha \in L$. 
Definition 2.7. [8] A map $N: X \rightarrow L^{L^{X}}$ is called an $L$-neighborhood system on $X$ with $N(x)=N_{x}$ if $N$ satisfies the following conditions

(N1) $N_{x}\left(1_{X}\right)=1$ and $N_{x}\left(0_{X}\right)=0$,

(N2) $N_{x}(\lambda) \leq \lambda(x)$ for all $\lambda \in L^{X}$,

(N3) If $\lambda \leq \mu$, then $N_{x}(\lambda) \leq N_{x}(\mu)$,

(N4) $N_{x}(\lambda \odot \mu) \geq N_{x}(\lambda) \odot N_{x}(\mu)$ for each $\lambda, \mu \in L^{X}$,

An $L$-neighborhood system is called stratified if

(R) $N_{x}(\alpha \odot \lambda) \geq \alpha \odot N_{x}(\lambda)$ for all $\lambda \in L^{X}$ and $\alpha \in L$.

The pair $(X, N)$ is called an $L$-neighborhood space.

Let $\left(X, N_{X}\right)$ and $\left(Y, N_{Y}\right)$ be two $L$-neighborhood spaces. A mapping $\phi$ : $X \rightarrow Y$ is said to be an $N$-map at $x \in X$ iff $\left(N_{Y}\right)_{\phi(x)}(\lambda) \leq\left(N_{X}\right)_{x}\left(\phi^{\leftarrow}(\lambda)\right)$ for each $\lambda \in L^{Y}$. $\phi$ is an $N$-map if it is an $N$-map at every $x \in X$.

\section{3. $L$-Neighborhood Systems and $L$-Fuzzy Topologies}

Theorem 3.1. Let $(X, \mathcal{T})$ be an $L$-fuzzy topological space. Define two maps $N^{\mathcal{T}}, M^{\mathcal{T}}: X \rightarrow L^{L^{X}}$ as follows:

$$
\begin{aligned}
N_{x}^{\mathcal{T}}(\lambda) & =\bigvee_{\mu \in L^{X}} \mathcal{T}(\mu) \odot S(\mu, \lambda) \odot \mu(x) \\
M_{x}^{\mathcal{T}}(\lambda) & =\bigvee_{\mu \in L^{X}}\{\mathcal{T}(\mu) \odot \mu(x) \mid \mu \leq \lambda\} .
\end{aligned}
$$

Then: (1) $\left(X, N^{\mathcal{T}}\right)$ is a stratified $L$-neighborhood space.

(2) $\left(X, M^{\mathcal{T}}\right)$ is an $L$-neighborhood space with $M^{\mathcal{T}} \leq N^{\mathcal{T}}$. Moreover, if $\mathcal{T}$ is enriched, $M^{\mathcal{T}}$ is stratified.

(3) If $\mathcal{T}$ is enriched, then $M^{\mathcal{T}}=N^{\mathcal{T}}$.

Proof. $(\mathrm{N} 1) N_{x}^{\mathcal{T}}\left(1_{X}\right)=\bigvee_{\mu \in L^{X}} \mathcal{T}(\mu) \odot S\left(\mu, 1_{X}\right) \odot \mu(x) \geq \mathcal{T}\left(1_{X}\right) \odot S\left(1_{X}, 1_{X}\right) \odot$ $1_{X}(x)=1$.

(N2) We have to show that $N_{x}^{\mathcal{T}}(\lambda) \leq \lambda(x)$ for each $\lambda \in L^{X}$ from:

$$
\begin{aligned}
& N_{x}^{\mathcal{T}}(\lambda)=\bigvee_{\mu \in L^{X}}(\mathcal{T}(\mu) \odot S(\mu, \lambda) \odot \mu(x)) \\
& \leq \bigvee_{\mu \in L^{X}}(\mathcal{T}(\mu) \odot(\mu(x) \rightarrow \lambda(x)) \odot \mu(x))
\end{aligned}
$$




$$
\leq \bigvee_{\mu \in L^{X}}(\mathcal{T}(\mu) \odot \lambda(x)) \leq \lambda(x)
$$

(N3)

$$
\begin{aligned}
& N_{x}^{\mathcal{T}}(\lambda)=\bigvee_{\rho \in L^{X}}(\mathcal{T}(\rho) \odot S(\rho, \lambda) \odot \rho(x)) \\
& \leq \bigvee_{\rho \in L^{X}}\left((\mathcal{T}(\rho) \odot S(\rho, \mu) \odot \rho(x))=N_{x}^{\mathcal{T}}(\mu)\right.
\end{aligned}
$$

(N4)

$$
\begin{aligned}
N_{x}^{\mathcal{T}}(\lambda) \odot N_{x}^{\mathcal{T}}(\mu) \\
=\bigvee_{\rho \in L^{X}}(\mathcal{T}(\rho) \odot S(\rho, \lambda) \odot \rho(x)) \odot \bigvee_{\nu \in L^{X}}(\mathcal{T}(\nu) \odot S(\nu, \mu) \odot \nu(x)) \\
=\bigvee_{\rho, \nu \in L^{X}}(\mathcal{T}(\rho) \odot \mathcal{T}(\nu) \odot S(\rho, \lambda) \odot S(\nu, \mu) \odot(\rho \odot \nu)(x)) \\
\leq \bigvee_{\rho, \nu \in L^{X}}(\mathcal{T}(\rho \odot \nu) \odot S(\rho \odot \nu, \lambda \odot \mu) \odot(\rho \odot \nu)(x))
\end{aligned}
$$

(by Lemma 2.4 )

$$
\leq \bigvee_{\gamma \in L^{X}}(\mathcal{T}(\gamma) \odot S(\gamma, \lambda \odot \mu) \odot \gamma(x))=N_{x}^{\mathcal{T}}(\lambda \odot \mu)
$$

For all $\lambda \in L^{X}$ and $\alpha \in L$, since $S(\rho, \alpha \odot \lambda) \geq \alpha \odot S(\rho, \lambda)$, we have

$$
\begin{aligned}
& N_{x}^{\mathcal{T}}(\alpha \odot \lambda)=\bigvee_{\rho \in L^{X}}(\mathcal{T}(\rho) \odot S(\rho, \lambda) \odot \rho(x)) \\
& \geq \bigvee_{\rho \in L^{X}}(\mathcal{T}(\rho) \odot \alpha \odot S(\rho, \lambda) \odot \rho(x)) \\
& =\alpha \odot \bigvee_{\rho \in L^{X}}(\mathcal{T}(\rho) \odot S(\rho, \lambda) \odot \rho(x))=\alpha \odot N_{x}^{\mathcal{T}}(\lambda) .
\end{aligned}
$$

(2) By (1), we similarly prove that $M^{\mathcal{T}}$ is an $L$-neighborhood system.

$$
M_{x}^{\mathcal{T}}(\lambda)=\bigvee_{\rho \leq \lambda}(\mathcal{T}(\rho) \odot S(\rho, \lambda) \odot \rho(x))
$$




$$
\leq \bigvee_{\rho \in L^{X}}(\mathcal{T}(\rho) \odot S(\rho, \lambda) \odot \rho(x))=N_{x}^{\mathcal{T}}(\lambda)
$$

If $\mathcal{T}$ is enriched, $M^{\mathcal{T}}$ is stratified from:

$$
\begin{aligned}
\alpha \odot M_{x}^{\mathcal{T}}(\lambda)=\alpha \odot \bigvee_{\rho \leq \lambda}(\mathcal{T}(\rho) \odot \rho(x)) \\
=\bigvee_{\rho \leq \lambda}(\mathcal{T}(\rho) \odot(\alpha \odot \rho)(x)) \\
\leq \bigvee_{\alpha \odot \rho \leq \alpha \odot \lambda}(\mathcal{T}(\alpha \odot \rho) \odot(\alpha \odot \rho)(x)) \\
=N_{x}^{\mathcal{T}}(\alpha \odot \lambda) .
\end{aligned}
$$

(3) Since $\mathcal{T}$ is enriched, then $\mathcal{T}(S(\rho, \lambda) \odot \rho) \geq \mathcal{T}(\rho)$. Thus

$$
\mathcal{T}(S(\rho, \lambda) \odot \rho) \odot S(\rho, \lambda) \odot \rho \geq \mathcal{T}(\rho) \odot S(\rho, \lambda) \odot \rho .
$$

Since $S(\rho, \lambda) \odot \rho \leq \lambda$, we have $M_{x}^{\mathcal{T}} \geq N_{x}^{\mathcal{T}}$.

Theorem 3.2. Let $(X, N)$ be an $L$-neighborhood space. Define a map $\mathcal{T}_{N}: L^{X} \rightarrow L$ by

$$
\mathcal{T}_{N}(\lambda)=\bigwedge_{x \in X}\left(\lambda(x) \rightarrow N_{x}(\lambda)\right)=S\left(\lambda, N_{-}(\lambda)\right) .
$$

Then

(1) $\mathcal{T}_{N}$ is an $L$-fuzzy topology on $X$,

(2) If $N$ is stratified, then $\mathcal{T}_{N}$ is an enriched $L$-fuzzy topology.

(3) If $N$ is stratified, $S(\lambda, \mu) \leq S\left(N_{-}(\lambda), N_{-}(\mu)\right)$ for all $\lambda, \mu \in L^{X}$.

(4) If $N$ is stratified, $N^{\mathcal{T}_{N}} \leq N$.

(5) If $N_{x}(\lambda) \leq \bigvee\left\{N_{x}(\mu) \mid \mu(y) \leq N_{y}(\lambda), \forall y \in X\right\}$, then $N^{\mathcal{T}_{N}} \geq N$.

(6) If $\mathcal{T}$ is an $L$-fuzzy topology on $X$, then $\mathcal{T}_{\mathcal{N}} \mathcal{T} \geq \mathcal{T}$.

(7) $\tau_{N}=\left\{\lambda \in L^{X} \mid \mathcal{T}_{N}(\lambda)=1\right\}$ is an $L$-topology on $X$,

(8) If $N$ is stratified, then $\tau_{N}$ is an enriched $L$-topology.

Proof. (1) (T1)

$$
\begin{aligned}
& \mathcal{T}_{N}\left(1_{X}\right)=\bigwedge_{x \in X}\left(1_{X}(x) \rightarrow N_{x}\left(1_{X}\right)\right)=1 \\
& \mathcal{T}_{N}\left(0_{X}\right)=\bigwedge_{x \in X}\left(0_{X}(x) \rightarrow N_{x}\left(0_{X}\right)\right)=1 .
\end{aligned}
$$


$(\mathrm{T} 2)$

$$
\begin{aligned}
& \mathcal{T}_{N}(\lambda \odot \mu) \\
& =\bigwedge_{x \in X}\left(\lambda(x) \odot \mu(x) \rightarrow N_{x}(\lambda \odot \mu)\right) \\
& \geq \bigwedge_{x \in X}\left(\lambda(x) \odot \mu(x) \rightarrow\left(N_{x}(\lambda) \odot N_{x}(\mu)\right)\right) \\
& \quad(\operatorname{by~Lemma~} 2.2(15)) \\
& \geq \bigwedge_{x \in X}\left(\lambda(x) \rightarrow N_{x}(\lambda)\right) \odot \bigwedge_{x \in X}\left(\mu(x) \rightarrow N_{x}(\mu)\right) \\
& =\mathcal{T}_{N}(\lambda) \odot \mathcal{T}_{N}(\mu) .
\end{aligned}
$$

(T3)

$$
\begin{aligned}
& \mathcal{T}_{N}\left(\bigvee_{i} \lambda_{i}\right)=\bigwedge_{x \in X}\left(\left(\bigvee_{i} \lambda_{i}(x) \rightarrow N_{x}\left(\bigvee_{i} \lambda_{i}\right)\right.\right. \\
& \geq \bigwedge_{x \in X}\left(\left(\bigvee _ { i } \left(\lambda_{i}(a)(x) \rightarrow \bigvee_{i} N_{x}\left(\lambda_{i}\right)\right.\right.\right. \\
& \quad(\text { by Lemma } 2.2(8)) \\
& \geq \bigwedge_{i} \bigwedge_{x \in X}\left(\lambda(x) \rightarrow N_{x}\left(\lambda_{i}\right)\right) \\
& =\bigwedge_{i} \mathcal{T}_{N}\left(\lambda_{i}\right)
\end{aligned}
$$

(2) By Lemma 2.2 (14), we have

$$
\begin{aligned}
& \mathcal{T}_{N}(\alpha \odot \lambda) \\
& =\bigwedge_{x \in X}\left((\alpha \odot \lambda)(x) \rightarrow N_{x}(\alpha \odot \lambda)\right) \\
& \geq \bigwedge_{x \in X}\left((\alpha \odot \lambda)(x) \rightarrow\left(\alpha \odot N_{x}(\lambda)\right)\right) \\
& \geq \bigwedge_{x \in X}\left(\lambda(x) \rightarrow N_{x}(\lambda)\right)=\mathcal{T}_{N}(\lambda) .
\end{aligned}
$$

(3) Since $N$ is stratified, $S(\lambda, \mu) \odot N_{x}(\lambda) \leq N_{x}(S(\lambda, \mu) \odot \lambda) \leq N_{x}(\mu)$. Thus, $S(\lambda, \mu) \leq N_{x}(\lambda) \rightarrow N_{x}(\mu)$.

(4)

$$
\begin{aligned}
N_{x}^{\mathcal{T}_{N}}(\lambda)=\bigvee_{\mu \in L^{X}}\left(\mathcal{T}_{N}(\mu) \odot S(\mu, \lambda) \odot \mu(x)\right) \\
=\bigvee_{\mu \in L^{X}}\left(S\left(\mu, N_{-}(\mu)\right) \odot S(\mu, \lambda) \odot \mu(x)\right) \\
\left.\quad \quad \text { by the definition of } \mathcal{T}_{N}\right) \\
\leq \bigvee_{\mu \in L^{X}} S\left(\mu, N_{-}(\mu)\right) \odot S\left(N_{-}(\mu), N_{-}(\lambda)\right) \odot \mu(x) \\
\leq \bigvee_{\mu \in L^{X}} S\left(\mu, N_{-}(\lambda)\right) \odot \mu(x)(\text { by Lemma } 2.4(5)) \\
\leq N_{x}(\lambda) .
\end{aligned}
$$


(5) Since $N_{x}(\lambda) \leq \bigvee\left\{N_{x}(\mu) \mid \mu(y) \leq N_{y}(\lambda), \forall y \in X\right\}$, we have $N_{x}(\lambda) \leq$ $N_{x}\left(N_{-}(\lambda)\right)$. By $(\mathrm{N} 2), N_{x}\left(N_{-}(\lambda)\right) \leq N_{x}(\lambda)$. Hence $N_{x}\left(N_{-}(\lambda)\right)=N_{x}(\lambda)$. Thus,

$$
\begin{aligned}
& N_{x}^{\mathcal{T}_{N}}(\lambda)=\bigvee_{\mu \in L^{X}}\left(\mathcal{T}_{N}(\mu) \odot S(\mu, \lambda) \odot \mu(x)\right) \\
& \geq S\left(N_{-}(\lambda), N_{-}\left(N_{-}(\lambda)\right)\right) \odot S\left(N_{-}(\lambda), \lambda\right) \odot N_{x}(\lambda)\left(\text { put } \mu=N_{-}(\lambda)\right) \\
& \geq N_{x}(\lambda) .
\end{aligned}
$$

(6)

$$
\begin{aligned}
& \mathcal{T}_{N_{\mathcal{T}}}(\lambda)=S\left(\lambda, N_{-}^{\mathcal{T}}(\lambda)\right) \\
& =\bigwedge_{x \in X}\left(\lambda(x) \rightarrow N_{x}^{\mathcal{T}}(\lambda)\right) \\
& =\bigwedge_{x \in X}\left(\lambda(x) \rightarrow \bigvee_{\mu \in L^{X}} \mathcal{T}(\mu) \odot S(\mu, \lambda) \odot \mu(x)\right) \\
& \geq \bigwedge_{x \in X}(\lambda(x) \rightarrow \mathcal{T}(\lambda) \odot S(\lambda, \lambda) \odot \lambda(x)) \\
& \geq \mathcal{T}(\lambda) \odot S(\lambda, \lambda)=\mathcal{T}(\lambda) .
\end{aligned}
$$

(7)and (8) are easily proved from (1) and (2).

Theorem 3.3. Let $(X, \tau)$ be an $L$-topological space. Define two maps $M^{\tau}, N^{\tau}: X \rightarrow L^{L^{X}}$ by

$$
\begin{gathered}
M_{x}^{\tau}(\lambda)=\bigvee\{\rho(x) \mid \rho \leq \lambda, \rho \in \tau\}, \\
N_{x}^{\tau}(\lambda)=\bigvee_{\rho \in \tau}(\rho(x) \odot S(\rho, \lambda)) .
\end{gathered}
$$

Then the following properties hold.

(1) $\left(X, M^{\tau}\right)$ is an $L$-neighborhood space satisfying

$$
M_{x}(\lambda) \leq \bigvee\left\{M_{x}(\mu) \mid \mu(y) \leq M_{y}(\lambda), \forall y \in X\right\} .
$$

(2) $\left(X, N^{\tau}\right)$ is a stratified $L$-neighborhood space. 
(3) $M^{\tau}=M^{\mathcal{T}_{\tau}}$ and $N^{\tau}=N^{\mathcal{T}_{\tau}}$ with an $L$-fuzzy topology as

$$
\mathcal{T}_{\tau}(\lambda)= \begin{cases}1, & \text { if } \lambda \in \tau, \\ 0, & \text { otherwise }\end{cases}
$$

(4) If $\tau$ is enriched, then $M^{\tau}$ is stratified and $M^{\tau}=N^{\tau}$.

(5) $\mathcal{T}_{M^{\tau}}$ and $\mathcal{T}_{N^{\tau}}$ are $L$-fuzzy topologies on $X$,

(6) If $\tau$ is enriched, then $\mathcal{T}_{M^{\tau}}=\mathcal{T}_{N^{\tau}}$ is an enriched $L$-fuzzy topology.

(7) $N^{\mathcal{T}^{\tau}} \leq N^{\tau}$ and $S(\lambda, \mu) \leq S\left(N_{-}^{\tau}(\lambda), N_{-}^{\tau}(\mu)\right)$ for all $\lambda, \mu \in L^{X}$.

(8) If $\tau$ is enriched, $N^{\mathcal{T}_{M^{\tau}}} \leq M^{\tau}$ and $S(\lambda, \mu) \leq S\left(M_{-}^{\tau}(\lambda), M_{-}^{\tau}(\mu)\right)$ for all $\lambda, \mu \in L^{X}$.

(9) $M^{\mathcal{T}_{M} \tau} \geq M^{\tau}$

Proof. (1) We easily prove that $\left(X, M^{\tau}\right)$ is an $L$-neighborhood space. Put $M_{-}^{\tau}(\lambda)=\bigvee\{\rho \mid \rho \leq \lambda, \rho \in \tau\}$ with $M_{-}^{\tau}(x)=M_{x}^{\tau}$. Then $M_{-}^{\tau}(\lambda) \in \tau$. By (N3) and the definition of $M^{\tau}$,

$$
\begin{gathered}
M_{x}^{\tau}\left(M_{-}^{\tau}(\lambda)\right)=M_{x}^{\tau}(\lambda) \\
M_{x}^{\tau}(\lambda)=M_{x}^{\tau}\left(M_{-}^{\tau}(\lambda)\right) \leq \bigvee\left\{M_{x}^{\tau}(\rho) \mid \rho(y) \leq M_{y}^{\tau}(\lambda)\right\}
\end{gathered}
$$

(2) It similarly proved as Theorem 3.1(1).

(3)

$$
\begin{aligned}
& M_{x}^{\tau}(\lambda)=\bigvee\{\rho(x) \mid \rho \leq \lambda, \rho \in \tau\}=\bigvee\left\{\mathcal{T}_{\tau}(\rho) \odot \rho(x) \mid \rho \leq \lambda\right\} \\
& N_{x}^{\tau}(\lambda)=\bigvee{ }_{\rho \in \tau}(\rho(x) \odot S(\rho, \lambda))=\bigvee\left(\mathcal{T}_{\tau}(\rho) \odot \rho(x) \odot S(\rho, \lambda)\right)
\end{aligned}
$$

$$
\begin{aligned}
& \alpha \odot M_{x}^{\tau}(\lambda)=\alpha \odot \bigvee\{\rho \mid \rho \leq \lambda, \rho \in \tau\} \\
& \leq \bigvee\{\alpha \odot \rho \mid \alpha \odot \rho \leq \alpha \odot \lambda, \alpha \odot \rho \in \tau\} \leq M_{x}^{\tau}(\alpha \odot \lambda)
\end{aligned}
$$

Let $\rho$ with $\rho \leq \lambda$ and $\rho \in \tau$. Then $\rho(x) \odot S(\rho, \lambda)=\rho(x) \odot 1=\rho(x)$. Thus $\rho(x) \leq N_{x}^{\tau}(\lambda)$. Therefore $M_{x}^{\tau}(\lambda) \leq N_{x}^{\tau}(\lambda)$.

Let $\rho(x) \odot S(\rho, \lambda)$ with $\rho \in \tau$. Since $\tau$ is enriched, $\rho \odot S(\rho, \lambda) \in \tau$ and $\rho(x) \odot S(\rho, \lambda) \leq \rho(x) \odot(\rho(x) \rightarrow \lambda(x)) \leq \lambda(x)$. Then $N_{x}^{\tau}(\lambda) \leq M_{x}^{\tau}(\lambda)$.

Other cases are easily proved from Theorem 3.2.

Theorem 3.4. Let $\left(X, \mathcal{T}_{X}\right)$ and $\left(Y, \mathcal{T}_{Y}\right)$ be $L$-fuzzy topological spaces. If a mapping $\phi:\left(X, \mathcal{T}_{X}\right) \rightarrow\left(Y, \mathcal{T}_{Y}\right)$ is $L$-fuzzy continuous, then $\phi:\left(X, N^{\mathcal{T}_{X}}\right) \rightarrow$ $\left(Y, N^{\mathcal{T}_{Y}}\right)$ is an $N$ - map and $\phi:\left(X, M^{\mathcal{T}_{X}}\right) \rightarrow\left(Y, M^{\mathcal{T}_{Y}}\right)$ is an $N$ - map. 
Proof.

$$
\begin{aligned}
& N_{\phi(x)}^{\mathcal{T}_{Y}}(\lambda)=\bigvee_{\mu \in L^{Y}} \mathcal{T}_{Y}(\mu) \odot S(\mu, \lambda) \odot \mu(\phi(x)) \\
& \leq \bigvee_{\mu \in L^{Y}}\left(\mathcal{T}_{X}\left(\phi^{\leftarrow}(\mu)\right) \odot S\left(\phi^{\leftarrow}(\mu), \phi^{\leftarrow}(\lambda)\right) \odot \phi^{\leftarrow}(\mu)(x)\right) \leq N_{x}^{\mathcal{T}_{X}}\left(\phi^{\leftarrow}(\lambda)\right) .
\end{aligned}
$$

$$
\begin{aligned}
& M_{\phi(x)}^{\mathcal{T}_{Y}}(\lambda)=\bigvee_{\mu \leq \lambda} \mathcal{T}_{Y}(\mu) \odot \mu(\phi(x)) \\
& \leq \bigvee_{\phi^{\leftarrow}(\mu) \leq \phi^{\leftarrow}(\lambda)}\left(\mathcal{T}_{X}\left(\phi^{\leftarrow}(\mu)\right) \odot \phi^{\leftarrow}(\mu)(x)\right) \leq M_{x}^{\mathcal{T}_{X}}\left(\phi^{\leftarrow}(\lambda)\right) .
\end{aligned}
$$

Theorem 3.5. Let $\left(X, N_{X}\right)$ and $\left(Y, N_{Y}\right)$ be $L$-neighborhood spaces. If a mapping $\phi:\left(X, N_{X}\right) \rightarrow\left(Y, N_{Y}\right)$ is an $N$-map, then $\phi:\left(X, \mathcal{T}_{N_{X}}\right) \rightarrow\left(Y, \mathcal{T}_{N_{Y}}\right)$ is $L$-fuzzy continuous.

Proof. Since $\left(N_{Y}\right)_{\phi(x)}(\lambda) \leq\left(N_{X}\right)_{x}\left(\phi^{\leftarrow}(\lambda)\right)$, we have

$$
\begin{aligned}
& \mathcal{T}_{N_{Y}}(\lambda)=\bigwedge_{y \in Y}\left(\lambda(y) \rightarrow\left(N_{Y}\right)_{y}(\lambda)\right) \\
& \leq \bigwedge_{x \in X}\left(\phi^{\leftarrow}(\lambda)(x) \rightarrow\left(N_{Y}\right)_{\phi(x)}(\lambda)\right) \\
& \leq \bigwedge_{x \in X}\left(\phi^{\leftarrow}(\lambda)(x) \rightarrow\left(N_{X}\right)_{x}\left(\phi^{\leftarrow}(\lambda)\right)\right)=\mathcal{T}_{N_{X}}\left(\phi^{\leftarrow}(\lambda)\right)
\end{aligned}
$$

Example 3.6. Let $(L=[0,1], \odot, \rightarrow)$ be a complete residuated lattice defined by

$$
x \odot y=(x+y-1) \vee 0, x \rightarrow y=(1-x+y) \wedge 1 .
$$

Let $X=\{x, y, z\}$ be a set and $\rho, \rho \odot \rho \in L^{X}$ such that

$$
\begin{gathered}
\rho(x)=0.5, \rho(y)=0.6, \rho(z)=0.6 \\
\rho \odot \rho(x)=0, \rho \odot \rho(y)=0.2, \rho \odot \rho(z)=0.2 .
\end{gathered}
$$


(1) We define an $L$-topology $\tau=\left\{0_{X}, 1_{X}, \rho, \rho \odot \rho\right\}$. We obtain two [0,1]neighborhood systems $N^{\tau}, M^{\tau}: X \rightarrow[0,1]^{[0,1]^{X}}$ as follows:

$$
\begin{gathered}
M_{x}^{\tau}(\lambda)=\left\{\begin{array}{ll}
1, & \text { if } \lambda=1_{X}, \\
0.5, & \text { if } \lambda \geq \rho, \\
0, & \text { otherwise. }
\end{array} \quad M_{y}^{\tau}(\lambda)= \begin{cases}1, & \text { if } \lambda=1_{X}, \\
0.6, & \text { if } \lambda \geq \rho, \\
0.2, & \text { if } \lambda \geq \rho \odot \rho, \\
& \lambda \geq \rho, \\
0, & \text { otherwise. }\end{cases} \right. \\
M_{z}^{\tau}(\lambda)= \begin{cases}1, & \text { if } \lambda=1_{X}, \\
0.6, & \text { if } \lambda \geq \rho, \\
0.2, & \text { if } \lambda \geq \rho \odot \rho, \lambda \geq \rho, \\
0, & \text { otherwise. }\end{cases} \\
N_{x}^{\tau}(\lambda)=S(1, \lambda) \vee(\rho(x) \odot S(\rho, \lambda)) \vee((\rho \odot \rho)(x) \odot S(\rho \odot \rho, \lambda)) .
\end{gathered}
$$

We obtain $L$-fuzzy topologies $\mathcal{T}_{M^{\tau}}, \mathcal{T}_{N^{\tau}}: L^{X} \rightarrow L$ as follows

$$
\begin{gathered}
\mathcal{T}_{M^{\tau}}(\lambda)= \begin{cases}1, & \text { if } \lambda=1_{X}, \lambda=0_{X}, \\
\wedge_{x \in X}(\lambda(x) \rightarrow \rho(x)), & \text { if } \lambda \geq \rho, \\
\wedge_{x \in X}(\lambda(x) \rightarrow(\rho \odot \rho)(x)), & \text { if } \lambda \geq \rho \odot \rho, \lambda \geq \rho, \\
\wedge_{x \in X}\left(\lambda(x) \rightarrow 0_{X}\right), & \text { otherwise. }\end{cases} \\
\mathcal{T}_{N^{\tau}}(\lambda)=\bigwedge_{x \in X}\left(\lambda(x) \rightarrow N_{x}^{\tau}(\lambda)\right) .
\end{gathered}
$$

Then $\lambda \in \tau$ iff $\mathcal{T}_{M^{\tau}}(\lambda)=1$. $\mathcal{T}_{M^{\tau}}$ is not enriched from:

$$
\begin{aligned}
& \mathcal{T}_{M^{\tau}}((0.1,0.1,0.1))=\mathcal{T}_{M^{\tau}}\left((0.1) \odot 1_{X}\right)=\wedge_{x \in X}\left((0.1,0.1,0.1) \rightarrow 0_{X}\right) \\
& =0.9 \nsupseteq \mathcal{T}_{M^{\tau}}\left(1_{X}\right)=1 .
\end{aligned}
$$

We have $\mathcal{T}_{N^{\tau}}((0.1,0.1,0.1))=1$ but $(0.1,0.1,0.1) \notin \tau$.

From Theorem 3.1, we obtain two [0,1]-neighborhood systems $M_{x}^{\mathcal{T}_{M}{ }^{\tau}}, N_{x}^{\mathcal{T}_{N} \tau}$ : $X \rightarrow[0,1]^{[0,1]^{X}}$ as follows:

$$
\begin{aligned}
& M_{x}^{\mathcal{T}_{M^{\tau}}}(\lambda)= \begin{cases}1, & \text { if } \lambda=1_{X}, \\
\bigvee_{\mu \leq \lambda}\left(\wedge_{x \in X}(\mu(x) \rightarrow \rho(x)) \odot \mu(x)\right), & \text { if } \lambda \geq \rho, \\
\bigvee_{\mu \leq \lambda}\left(\wedge_{x \in X}(\mu(x) \rightarrow(\rho \odot \rho)(x)) \odot \mu(x)\right), & \text { if } \lambda \geq \rho \odot \rho, \\
\bigvee_{\mu \leq \lambda}\left(\wedge_{x \in X}(\mu(x) \rightarrow 0) \odot \mu(x)\right)=0, & \lambda \geq \rho,\end{cases} \\
& N_{x}^{\mathcal{T}_{N^{\tau}}}(\lambda)=\bigvee_{\mu \in L^{X}} \mathcal{T}_{N^{\tau}}(\mu) \odot S(\mu, \lambda) \odot \mu(x)
\end{aligned}
$$


Then, if $\lambda \geq \rho$, since $\bigvee_{\mu \leq \lambda}\left(\wedge_{x \in X}(\mu(x) \rightarrow \rho(x)) \odot \mu(x)\right) \geq\left(\wedge_{x \in X}(\rho(x) \rightarrow\right.$ $\rho(x)) \odot \rho(x))=\rho(x), M_{x}^{\overline{\mathcal{T}}_{M} \tau}(\lambda) \geq M_{x}^{\tau}(\lambda)$. Other case, we similarly prove $M_{x}^{\mathcal{T}_{M \tau}}(\lambda) \geq M_{x}^{\tau}(\lambda)$.

If $\lambda \geq \rho, N_{x}^{\mathcal{T}^{\tau} \tau}(\lambda) \geq \mathcal{T}_{N^{\tau}}(\rho) \odot S(\rho, \lambda) \odot \rho(x)=\rho=N_{x}^{\tau}(\lambda)$.

If $\lambda \geq \rho \odot \rho, N_{x}^{\mathcal{T}_{N^{\tau}}}(\lambda) \geq \mathcal{T}_{N^{\tau}}(\rho \odot \rho) \odot S(\rho \odot \rho, \lambda) \odot(\rho \odot \rho)(x)=(\rho \odot \rho)(x)=$ $N_{x}^{\tau}(\lambda)$. Hence $N_{x}^{\mathcal{T}_{N \tau}}(\lambda) \geq N_{x}^{\tau}(\lambda)$.

(2) Let $\omega, \omega \odot \omega \in[0,1]^{X}$ such that

$$
\begin{gathered}
\omega(x)=0.1, \omega(y)=0.8, \omega(z)=0.7, \\
\omega \odot \omega(x)=0, \omega \odot \omega(y)=0.6, \omega \odot \omega(z)=0.4 .
\end{gathered}
$$

We define an $[0,1]$-fuzzy topology $\mathcal{T}:[0,1]^{X} \rightarrow[0,1]$ as follows

$$
\mathcal{T}(\lambda)= \begin{cases}1, & \text { if } \lambda=1_{X}, \lambda=0_{X}, \\ 0.6, & \text { if } \lambda=\omega \\ 0.3, & \text { if } \lambda=\omega \odot \omega \\ 0, & \text { otherwise. }\end{cases}
$$

We obtain two $[0,1]$-neighborhood systems $M_{-}^{\mathcal{T}}, N_{-}^{\mathcal{T}}: X \rightarrow[0,1]^{[0,1]^{X}}$ as follows

$$
\begin{aligned}
& M_{-}^{\mathcal{T}}(\lambda)= \begin{cases}1, & \text { if } \lambda=1_{X}, \\
(0.6 \odot \omega(-)) \vee(0.3 \odot(\omega \odot \omega)(-)), & \text { if } 1_{X} \neq \lambda \geq \omega, \\
0.3 \odot(\omega \odot \omega)(-), & \text { if } \lambda \geq \omega \odot \omega, \\
& \lambda \geq \omega, \\
0, & \text { otherwise. }\end{cases} \\
& N_{-}^{\mathcal{T}}(\lambda)=\bigvee_{\mu \in L^{X}}(\mathcal{T}(\mu) \odot S(\mu, \lambda) \odot \mu(-)) \\
& =S\left(1_{X}, \lambda\right) \vee(0.6 \odot S(\omega, \lambda) \odot \omega(-)) \\
& \vee(0.3 \odot S(\omega \odot \omega, \lambda) \odot(\omega \odot \omega)(-)) \text {. }
\end{aligned}
$$

For $\lambda_{1}=(0.9,0.4,0.2)$ and $\lambda_{2}=(0,1,1)$,

$$
\begin{gathered}
M_{-\mathcal{T}}^{\mathcal{T}}\left(\lambda_{1}\right)=(0,0,0), \\
M_{-}^{\mathcal{T}}\left(\lambda_{2}\right)=0.3 \odot(\omega \odot \omega)(-)=(0,0,0), \\
N_{-}^{\mathcal{T}}\left(\lambda_{1}\right)=\left(0.2 \odot 1_{X}\right) \vee(0.6 \odot 0.5 \odot \omega(-)) \\
\\
N_{-}^{\mathcal{T}}\left(\lambda_{2}\right) \quad=(0.3 \odot 0.8 \odot(\omega \odot \omega)(-))=(0.2,0.2,0.2), \\
\\
\quad \vee(0.3 \odot 1 \odot(\omega \odot \omega)(-))=(0,0.3,0.2) .
\end{gathered}
$$




\section{References}

[1] R. Bělohlávek, Fuzzy Relational Systems, Kluwer Academic Publishers, New York, 2002.

[2] C.L. Chang, Fuzzy topological spaces, J. Math. Anal. Appl., 24 (1968), 182-190.

[3] D. Čimoka, A.Šostak, L-fuzzy syntopogenous structures, Part I: Fundamentals and application to $L$-fuzzy topologies, $L$-fuzzy proximities and $L$-fuzzy uniformities, Fuzzy Sets and Systems, 232 (2013), 74-97, doi: 10.1016/j.fss2013.04.009.

[4] J. Fang, I-fuzzy Alexandrov topologies and specialization orders, Fuzzy Sets and Systems, 158(2007) 2359-2374, doi: 10.1016/j.fss2007.05.001.

[5] J. Fang, The relationship between $L$-ordered convergence structures and strong $L$ topologies, Fuzzy Sets and Systems, 161 (2010), 2923-2944, doi: 10.1016/j.fss2010.05.006.

[6] P. Hájek, Metamathematices of Fuzzy Logic, Kluwer Academic Publishers, Dordrecht, 1998, doi: 10.1007/978-94-011-5300-3.

[7] U. Höhle, S.E. Rodabaugh, Mathematics of Fuzzy Sets: Logic, Topology, and Measure Theory, The Handbooks of Fuzzy Sets Series 3, Kluwer Academic Publishers, Boston, 1999, doi: 10.1007/978-1-4615-5079-2.

[8] R. Lowen, Fuzzy neighborhood spaces, Fuzzy Sets and Systems, 7 (1982), 165-189.

[9] H. Lai, D. Zhang, Fuzzy preorder and fuzzy topology, Fuzzy Sets and Systems, 157 (2006), 1865-1885, doi: 10.1016/j.fss2006.02.013.

[10] A.A. Ramadan, E.H. Elkordy, Y.C.Kim, L-neighborhood systems, L-topology and Lquasi Uniformities, J. Math. Comput. Sci., 5, No. 1 (2015), 11-24.

[11] A.A. Ramadan, E.H.Elkordy, Y.C. Kim, Relationships between $L$-fuzzy quasi-uniform stuctures and L-fuzzy topologies, Journal of Intelligent and Fuzzy Systems, 28 (2015), 2319-2327, doi: 10.3233/IFS-141515.

[12] S.E. Rodabaugh, E.P. Klement, Topological and Algebraic Structures In Fuzzy Sets, The Handbook of Recent Developments in the Mathematics of Fuzzy Sets, Kluwer Academic Publishers, Boston, Dordrecht, London, 2003. 
